\title{
Construction of Second Order Rotatable Simplex Designs
}

\author{
Otieno-Roche Emily ${ }^{1}$, Koske Joseph ${ }^{2}$, Mutiso John ${ }^{2}$ \\ ${ }^{1}$ Department of Computer and Information Technology, Africa Nazarene University, Nairobi, Kenya \\ ${ }^{2}$ Department of Statistics and Computer Science, Moi University, Eldoret, Kenya
}

\section{Email addresses:}

rocheemmy@gmail.com (Otieno-Roche E.), koske4@yahoo.co.uk (Koske J.), johnkasome@yahoo.com (Mutiso J.)

\section{To cite this article:}

Otieno-Roche Emily, Koske Joseph, Mutiso John. Construction of Second Order Rotatable Simplex Designs. American Journal of Theoretical and Applied Statistics. Vol. 6, No. 6, 2017, pp. 297-302. doi: 10.11648/j.ajtas.20170606.16

Received: June 2, 2017; Accepted: June 16, 2017; Published: December 7, 2017

\begin{abstract}
Rotatable designs are mainly for the exploration of response surfaces. These designs provide the preferred property of constant prediction variance at all points that are equidistant from the design center, thus improving the quality of the prediction. Initially, they were constructed through geometrical configurations and several second order designs were obtained. Full Factorial Design of Experiment provides the most response information about factor main effects and interactions, the process model's coefficients for all factors and interactions, and when validated, allows process to be optimized. On the other hand, mixture designs are a special case of response surface designs where prediction and optimization are the main goals. These designs usually predict all possible formulations of the ingredients however, little or no research has been done incorporating rotatability with the mixture designs. This paper therefore aims at constructing Rotatable Simplex Designs (RSDs) using the properties of Simplex - Lattice Designs (SLDs) in connection with Full Factorial Designs (FFDs).
\end{abstract}

Keywords: Response Surface Designs, Second-Order Rotatable Designs (SORD), Mixture Designs, Moment Matrices, Central Composite Designs

\section{Introduction}

A response surface design is an experimental field in which treatments are various combinations of different levels of the factors that are quantitative. Here the main objective of the experimenter is usually to estimate the absolute response or the parameters of a model providing the relationship between the response and the factors. In many experimental situations this relationship is a functional one say $Y=$ $f\left(t_{1}, t_{2}, \ldots, t_{k}\right)+e$ where $Y$ is the response, $t_{1}, t_{2}, \ldots, t_{k}$ are $k$ factors and $e$ is the random error term.

A $k-$ simplex is a generalization of the notion of a $k-$ dimensional polytope which is the convex hull of its $k+$ 1 vertices. Simplex Designs are used to study the effects of mixture components on the response variable. There are standard mixture designs for fitting standard models such as Simplex - Lattice Designs and Simplex - Centroid Designs

In this paper, the properties of Simplex Lattice Designs, in connection with Full Factorial Designs have been used to construct Second Order Rotatable Simplex Design with any number of factors.

\section{Second Order Rotatable Designs}

Box and Hunter (1957) introduced rotatability as a natural and highly desirable property in response surface methodology for the exploration of response surfaces. They constructed these designs through geometrical configurations and obtained second order rotatable designs (SORD). Das and Narasimham (1962) constructed second order rotatable designs (SORD) through balanced incomplete block designs (BIBD). Tyagi (1964) constructed SORD using pairwise balanced designs (PBD). Panda and Das (1994) studied first order rotatable designs with correlated errors. Das (1997, 1999) introduced robust second order rotatable designs (RSORD). Rajyalakshmi and Victorbabu (2011) constructed robust rotatable central composite designs (RRCCD) for factors $2 \leq v \leq 17$. Victorbabu and Rajyalakshmi (2012) gave a new method of construction of robust second order rotatable designs using BIBD.

Victorbabu and Rajyalakshmi (2012) studied robust slope rotatable central composite designs (RSRCCD) for the factors $2 \leq v \leq 8$. Further, Victorbabu and Rajyalakshmi 
(2012) studied robust slope rotatable designs (RSOSRD) using BIBD for the factors $3 \leq v \leq 8$.

Rajyalakshmi and Victorbabu (2014) suggested an empirical study of second order rotatable designs under tridiagonal correlation structure of errors using central composite designs.

\section{Conditions for Second - Order Rotatability}

Rotatable designs provide the preferred property of constant prediction variance at all points that are equidistant from the design center, thus improving the quality of the prediction.

$$
y_{u}=\beta_{o u}+\sum_{i u} \beta_{i u} t_{i u}+\sum_{i u} \beta_{i i u} t_{i u}^{2}+\sum_{i u \leq j u} \beta_{i j u} t_{i u} t_{j u}+\sum_{i u \leq j u \leq k u} \beta_{i j k u} t_{i u} t_{j u} t_{k u}+\cdots+\varepsilon_{u}
$$

of order $d$ of the response $y$ as obtained from the treatments on the variates $t_{i}, i=1,2, \cdots, k$, with some suitable origin and scale, can be so fitted that the variance function

$$
\operatorname{Var}(\hat{y})=t_{s}^{\prime}\left(T^{\prime} T\right)^{-1} t_{s} \sigma^{2}
$$

is a function of only $r_{u}^{2}=t_{1 u}^{2}+t_{2 u}^{2}+\cdots+t_{k u}^{2}$ i.e. the variance of $\hat{y}_{u}$ is a function of only the distance of $t$ from the origin not of the direction.

When the response surface is of the second degree (i.e. $d=2$ ), such constancy of variance is possible if the design points are selected to satisfy the following relations

$$
\begin{gathered}
\sum t_{i u}=0, \sum t_{i u} t_{j u}=0, \sum t_{i u} t_{j u}^{2}=0, \sum t_{i u}^{3}=0, \sum t_{i u} t_{j u}^{3}=0, \sum t_{i u} t_{j u} t_{k u}=0, \sum t_{i u} t_{j u} t_{k u}^{2}=0, \sum t_{i u} t_{j u} t_{k u} t_{l u}=0 \text { for } i \neq j \neq k \neq l . \\
\sum t_{i u}^{2}=\text { constant }=N \lambda_{2} \text { and } \sum t_{i u}^{4}=\text { constant }=3 N \lambda_{4} \text { for all } i \\
\sum t_{i u}^{2} t_{j u}^{2}=\text { constant for all } i \neq j \\
\sum_{i=1}^{k} t_{i u}=1
\end{gathered}
$$$$
\sum t_{i u}^{4}=3 \sum t_{i u}^{2} t_{j u}^{2} \text { for all } i \neq j
$$

where the summation in the above relations is over the design points $u=1,2, \ldots, N$.

\section{Moment Matrix}

The moment matrix

$$
M(T)=\sum_{j=1}^{k} w_{j} f\left(t_{j}\right) f\left(t_{j}\right)^{\prime}=\int_{T} f(t) f(t)^{\prime} d T
$$

reflects the statistical properties of a design $\mathrm{T}$.

\section{Simplex Designs}

A mixture experiment involves the study of performance of various mixtures formed by mixing two or more components called factors. The special nature of the factors necessitates specific regression models and specific types of experimental designs. Let $t_{i}$ represent the proportion of $i^{t h}$ factor in a mixture. Then the key feature of these factors is:

$$
0 \leq t_{i u} \leq 1 i=1,2, \ldots, k
$$

and
Simplex designs are used to study the effects of mixture components on the response variable. A $k-$ simplex is a generalization of the notion of a $k$-dimensional polytope which is the convex hull of its $k+1$ vertices.

Scheffe $(1958,1963)$ introduced Simplex Lattice Designs and Simplex Centroid Designs for estimation of the parameters in the mean response function.

The "standard" mixture designs suggested ensure:

(1) full column rank condition;

(2) general structure for the information matrix for routine computation of the variance and covariance of the estimates of the model parameters.

\section{Rotatable Simplex Design}

This design was constructed in connection with the Full Factorial design. Construction was done for two and three factors, followed by a generalization to $k$ factors.

Equation [5] from the SLD was used to generate the original design points for the RSD. The levels of the SLD were increased by taking all the combination levels of the original points from the SLD to conform to equation [3a] for rotatability. These points were then augmented with all the combination levels of the distance from the centre point $(a)$. 
Equation [3d] was then solved to attain rotatability.

\subsection{Rotatable Simplex Design (RSD) $\{k, 2\}$}

A RSD design for $k$ factors involves all possible formulations of $\{k, 2\}$, i.e the $k$ individual factor proportions belonging to the set $\pm\left\{0, \frac{1}{2}, 1\right\}$, augmented with the $2^{k}$ Full Factorial Design points of the distance from the centre.

\section{2. $R S D\{2,2\}$}

The design points which constitute a $2^{\text {nd }}$ order RSD are given by $\{2,2\} \cup \pm a$ as shown below:

Table 1. Design points for $R S D\{2,2\}$.
At the $u^{\text {th }}$ observation. $(u=1,2, \ldots, N=12)$

The value of $a$ is determined such that the rotatability, relation in $[3 d]$ is satisfied. Therefore using [Table 1] in $[3 d]$, the following relation is obtained

$$
2+4\left(\frac{1}{2}\right)^{4}+4 a^{4}=12\left(\frac{1}{2}\right)^{4}+12 a^{4}
$$

This then gives:

$$
a=\left[\frac{3}{16}\right]^{\frac{1}{4}}
$$

Following [1], the model used was

\begin{tabular}{ccccccccccccc}
\hline$t_{1 u}$ & 1 & -1 & 0 & 0 & $1 / 2$ & $-1 / 2$ & $-1 / 2$ & $1 / 2$ & $a$ & $-a$ & $a$ & $-a$ \\
$t_{2 u}$ & 0 & 0 & 1 & -1 & $1 / 2$ & $-1 / 2$ & $1 / 2$ & $-1 / 2$ & $a$ & $a$ & $-a$ & $-a$ \\
\hline
\end{tabular}

$$
y_{u}=\beta_{o u} t_{o u}+\beta_{1 u} t_{1 u}+\beta_{2 u} t_{2 u}+\beta_{11 u} t_{1 u}^{2}+\beta_{22 u} t_{2 u}^{2}+\beta_{12 u} t_{1 u} t_{2 u}+\varepsilon_{u}
$$

for the $u^{\text {th }}$ observation, $(u=1,2, \ldots, N=12)$.

Hence from [Table 1], the design matrix $T$ is arranged as:

Table 2. Design matrix $T$ for $\{2,2\} R S D$

\begin{tabular}{cccccccccccccccccccccccccccc}
\hline$t_{1 u}$ & 1 & -1 & 0 & 0 & 0 & 0 & $\frac{1}{2}$ & $-\frac{1}{2}$ & $\frac{1}{2}$ & $-\frac{1}{2}$ & $\frac{1}{2}$ & $-\frac{1}{2}$ & $\frac{1}{2}$ & $-\frac{1}{2}$ & 0 & 0 & 0 & 0 & 1 \\
$t_{2 u}$ & 0 & 0 & 1 & -1 & 0 & 0 & $\frac{1}{2}$ & $\frac{1}{2}$ & $-\frac{1}{2}$ & $-\frac{1}{2}$ & 0 & 0 & 0 & 0 & $\frac{1}{2}$ & $-\frac{1}{2}$ & $\frac{1}{2}$ & $-\frac{1}{2}$ \\
$t_{3 u}$ & 0 & 0 & 0 & 0 & 1 & -1 & 0 & 0 & 0 & 0 & $\frac{1}{2}$ & $\frac{1}{2}$ & $-\frac{1}{2}$ & $-\frac{1}{2}$ & $\frac{1}{2}$ & $\frac{1}{2}$ & $-\frac{1}{2}$ & $-\frac{1}{2}$ \\
\hline
\end{tabular}

$$
T=\left[\begin{array}{cccccc}
1 & 1 & 0 & 1 & 0 & 0 \\
1 & 1 & 0 & -1 & 0 & 0 \\
1 & 0 & 1 & 0 & 1 & 0 \\
1 & 0 & 1 & 0 & -1 & 0 \\
1 & \frac{1}{4} & \frac{1}{4} & \frac{1}{2} & \frac{1}{2} & \frac{1}{4} \\
& t_{1 u}^{2} & t_{2 u}^{2} & t_{1 u} & t_{2 u} & t_{i u} t_{2 u} \\
1 & \frac{1}{4} & \frac{1}{4} & -\frac{1}{2} & -\frac{1}{2} & \frac{1}{4} \\
& 1 & 1 & 1 & 1 & 1 \\
1 & \frac{1}{4} & \frac{1}{4} & -\frac{1}{2} & \frac{1}{2} & -\frac{1}{4} \\
1 & \frac{1}{4} & \frac{1}{4} & \frac{1}{2} & -\frac{1}{2} & -\frac{1}{4} \\
1 & a^{2} & a^{2} & a & a & a^{2} \\
1 & a^{2} & a^{2} & -a & a & -a^{2} \\
1 & a^{2} & a^{2} & a & -a & -a^{2} \\
1 & a^{2} & a^{2} & -a & -a & a^{2}
\end{array}\right]
$$

Now using [Table 2] in [4], the moment matrix is given as:

$$
\frac{1}{12}\left[\begin{array}{cccc}
12 & \left(3+4 a^{2}\right) 1_{2}^{\prime} & 0 & 0 \\
\left(3+4 a^{2}\right) 1_{2} & \left(\frac{9}{4}+4 a^{4}\right) \mathrm{I}_{2}+\left(\frac{1}{4}+4 a^{4}\right)\left(e_{1} e_{2}^{\prime}+e_{2} e_{1}^{\prime}\right) & 0 & 0 \\
0 & 0 & \left(3+4 a^{2}\right) \mathrm{I}_{2} & 0 \\
0 & 0 & 0 & \frac{1}{4}+4 a^{4}
\end{array}\right]
$$

Where $a$ is as given in [6]

\section{3. $\operatorname{RSD}\{3,2\}$}

Similarly, a $2^{\text {nd }}$ order $\{3,2\}$ RSD gives all the possible formulations as: 
Table 3. Design points for $R S D\{3,2\}$.

\begin{tabular}{|c|c|c|c|c|c|c|c|c|}
\hline$t_{1 u}$ & $-a$ & $-a$ & $-a$ & $-a$ & $a$ & $a$ & $a$ & $a$ \\
\hline$t_{2 u}$ & $-a$ & $-a$ & $a$ & $a$ & $-a$ & $-a$ & $a$ & $a$ \\
\hline$t_{3 u}$ & $-a$ & $a$ & $-a$ & $a$ & $-a$ & $a$ & $-a$ & $a$ \\
\hline
\end{tabular}

At the $u^{\text {th }}$ observation. $(u=1,2, \ldots, N=26)$

The choice of the design points in [Table 3] to satisfy the rotatability relation in [3d] yields:

$$
2+8\left(\frac{1}{2}\right)^{4}+8 a^{4}=12\left(\frac{1}{2}\right)^{4}+24 a^{4}
$$

This then gives:

$$
a=\left[\frac{7}{64}\right]^{\frac{1}{4}}
$$

Following [1], the model used was

$$
y_{u}=\beta_{o u} t_{o u}+\beta_{1 u} t_{1 u}+\beta_{2 u} t_{2 u}+\beta_{3 u} t_{3 u}+\beta_{11 u} t_{1 u}^{2}+\beta_{22 u} t_{2 u}^{2}+\beta_{33 u} t_{3 u}^{2}+\beta_{12 u} t_{1 u} t_{2 u}+\beta_{13 u} t_{1 u} t_{3 u}+\beta_{23 u} t_{2 u} t_{3 u}+\varepsilon_{u}
$$

for the $u^{\text {th }}$ observation, $(u=1,2, \ldots, N=26)$

[4] is:

The design matrix $T$ was then arranged as: (APPENDIX I), and the corresponding moment matrix for the design matrix as in

$$
\frac{1}{26}\left[\begin{array}{cccc}
26 & \left(4+8 a^{2}\right) 1_{3}^{\prime} & 0 & 0 \\
\left(4+8 a^{2}\right) 1_{3} & \left.\frac{9}{4} I_{3}+\left(\frac{1}{4}+8 a^{4}\right) J_{3}\right) & 0 & 0 \\
0 & 0 & \left(4+8 a^{2}\right) I_{3} & 0 \\
0 & 0 & 0 & \left(\frac{1}{4}+8 a^{4}\right) I_{3}
\end{array}\right]
$$

Where $a$ is as defined in [8].

\subsection{General RSD $\{k, 2\}$}

The above findings were generalized for a $\{k, 2\} \mathrm{RSD}$, and the rotatability conditions too considered obtaining;

$$
a=\left[\frac{k(k-1)+1}{2^{2 k}}\right]^{\frac{1}{4}}
$$

From [7] and [9] above, the generalized moment matrix for a $\{k, 2\}$ Rotatable Simplex Design is given as:

$$
\mathcal{M}=\frac{1}{N}\left(\begin{array}{ccc}
M_{1} & 0 & 0 \\
0 & M_{2} & 0 \\
0 & 0 & M_{3}
\end{array}\right)
$$

Where

$$
\mathrm{M}_{1}=\left(\begin{array}{cc}
N & b 1_{2}^{\prime} \\
b 1_{2} & c \mathrm{I}_{2}+d\left(e_{1} e_{2}^{\prime}+e_{2} e_{1}^{\prime}\right)
\end{array}\right), \quad \mathrm{M}_{2}=\mathrm{bI}_{\mathrm{k}} \quad \text { and } \mathrm{M}_{3}=\mathrm{dI}_{\frac{\mathrm{k}(\mathrm{k}-1)}{2}}
$$

In which

$$
\mathrm{N}=2\left(\mathrm{k}^{\mathrm{k}-1}+4\right), \quad \mathrm{b}=(\mathrm{k}+1)+2^{\mathrm{k}} \mathrm{a}^{2}, \quad \mathrm{c}=\frac{2^{3}+(\mathrm{k}-1)}{4}+2^{\mathrm{k}} \mathrm{a}^{4} \text { and } \quad \mathrm{d}=\frac{1}{4}+2^{\mathrm{k}} \mathrm{a}^{4}
$$

\section{Conclusion}

Rotatable designs have been widely studied. In recent years more emphasis has been placed by the chemical and processing field for finding regions where there is an improvement in response instead of finding the optimum response (Myers, Khuri, and Carter 1989). In practice, the experimenter aims at obtaining optimal designs with minimum cost.
When experimentation is expensive, difficult or time consuming, rotatable designs seem more appropriate in practice. One of the aims of design of experiment in general is to extract as much as possible information from a limited set of experimental study.

The newly suggested second order rotatable simplex design may add to the list of designs that seek to address the highlighted areas, and are very efficient in providing much information on experiment variable effects and overall 
experimental error in a minimum number of required runs.

\section{Appendix}

I. Design matrix $T$ for $\{3,2\}$ RSD

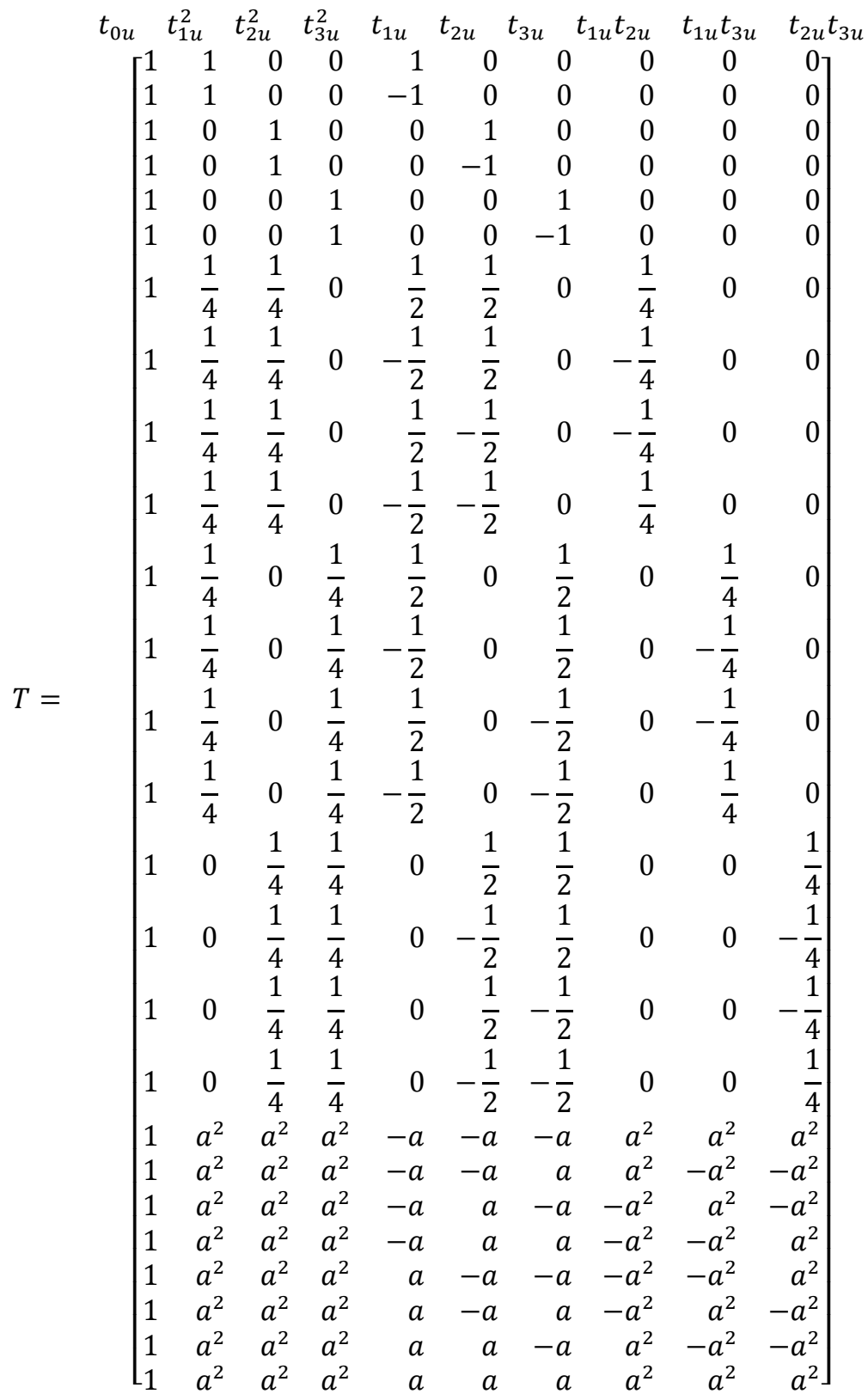

\section{References}

[1] Box, G. E. P., \& Draper, N. R. (1959). A basis for the selection of a response surface design. Journal of American Statistical Association, 54, 622-654.

[2] Das, M. N., \& Narasimham, V. L. (1962). Construction of rotatable designs through balanced incomplete block designs. Annals of Mathematical Statistics, 33(4), 1421-1439.

[3] Das, R. N. (1997). Robust second order rotatable designs: Part I RSORD. Calcutta Statistical Association Bulletin, 47, 199214.
[4] Das, R. N. (1999). Robust Second Order Rotatable Designs: Part II RSORD. Calcutta Statistical Association Bulletin, 49, 65-76.

[5] Myers, R., Khuri, A., \& Carter, W. (1989). Response Surface Methodology: 1966-1988. Technometrics, 31(2), 137-157. doi: $10.2307 / 1268813$.

[6] Panda, R. N., \& Das, R. N. (1994). First order rotatable designs with correlated errors. Calcutta Statistical Association Bulletin, 44, 83-101.

[7] Rajyalakshmi, K., \& Victorbabu B. R. (2014). Construction of second order rotatable designs under tri-diagonal correlation structure of errors using central composite designs. Journal of Statistics: Advances in Theory and Applications, 11(2), 71-90. 
[8] Rajyalakshmi, K., \& Victorbabu, B. R. (2011). Robust Second Order Rotatable Central Composite Designs. JP Journal of Fundamental and Applied Statistics, 1(2), 85-102.

[9] Tyagi, B. N. (1964). Construction of second order and third order rotatable designs through pairwise balanced designs and doubly balanced designs. Calcutta Statistical Association Bulletin, 13, 150-162.
[10] Victorbabu, B. R., \& Rajyalakshmi, K. (2012). A new method of construction of robust second order rotatable designs using balanced incomplete block designs. Open Journal of Statistics, 2(2), 88-96.

[11] Victorbabu, B. R., \& Rajyalakshmi, K. (2012). Robust second order slope rotatable designs using balanced incomplete block designs. Open Journal of Statistics, 2(2), 65-77. 